\title{
Immunological assessment of SARS-CoV-2 Infection in Pregnancy From Diagnosis to Delivery: A multicentre prospective observational study
}

Kate Glennon ${ }^{1}$, Jennifer Donnelly ${ }^{2}$, Susan Knowles ${ }^{3}$, Fionnuala McAuliffe ${ }^{4}$, Alma O Reilly $^{2}$, Siobhan Corcoran ${ }^{3}$, Jennifer Walsh ${ }^{3}$, Roger McMorrow ${ }^{3}$, Tess Higgins ${ }^{3}$, Lucy Bolger $^{3}$, Susan Clinton ${ }^{3}$, Sarah O Riordan ${ }^{3}$, Alex Start ${ }^{4}$, Doireann Roche ${ }^{3}$, Helena Bartels ${ }^{5}$, Ciara Malone $^{5}$, Karl McAuley ${ }^{6}$, Anthony Mc Dermott ${ }^{6}$, Rosanna Inzitari ${ }^{6}$, Colm P.F. O Donnell $^{7}$, Fergal Malone ${ }^{2}$, Shane Higgins ${ }^{3}$, Cillian De Gascun ${ }^{8}$, Peter Doran ${ }^{6}$, and Donal Brennan $^{1}$

${ }^{1}$ University College Dublin

${ }^{2}$ RCSI School of Medicine

${ }^{3}$ National Maternity Hospital

${ }^{4}$ UCD Perinatal Research Centre

${ }^{5}$ Rotunda Hospital

${ }^{6}$ Clinical Research Centre, UCD School of Medicine

${ }^{7}$ Neonatal Unit, UCD School of Medicine

${ }^{8}$ National Virus Reference Laboratory

November 16, 2020

\begin{abstract}
Objective We profile the maternal and fetal response to SARS-CoV-2 infection in symptomatic and asymptomatic pregnant women and make an assessment of passive immunity to the neonate, Design Multicentre prospective study. Setting Dublin, Ireland Methods RT-PCR for viral RNA via a nasopharyngeal swab was performed using the Cobas SARS-CoV-2 6800 platform. Maternal, and fetal serological antibody response, via umbilical cord bloods, was measured using both the Elecsys $\mathbb{R}$ immunoassay, Abbott SARS-CoV-2 IgG Assay and the IgM Architect assay. Main outcome Measure Prevalence of RT PCR positive SARS-CoV-2. Assessment of IgM and IgG anti-SARS-CoV-2 serology antibodies. Results Ten of twenty three symptomatic women had SARS-CoV-2 RNA in a nasopharyngeal swab. Five $(5 / 23,21.7 \%)$ demonstrated serological evidence of anti-SARS-CoV-2 IgG antibodies and seven $(30.4 \%, 7 / 23)$ were positive for IgM antibodies. In the asymptomatic cohort, the prevalence of SARS-CoV-2 infection in RNA was 0.16\% (1/608). IgG SARS-CoV-2 antibodies were detected in 1.67\% (10/598, 95\% CI $0 \cdot 8 \%-3 \cdot 1 \%)$ and IgM in 3.51\% (21/598, 95\% CI 2.3-5.5\%). Nine women had repeat testing between post baseline. Four $(4 / 9,44 \%)$ remained IgM positive, one IgG positive. IgG anti SARS-CoV-2 antibodies were detectable in cord bloods from babies born to five seropositive women who delivered during the study. Conclusion Using two independent serological assays, we present a comprehensive illustration of the antibody response to SARS-CoV-2 in pregnancy, and show a low prevalence of asymptomatic SARS-CoV-2. Transplacental migration of anti-SARS-CoV-2 antibodies was identified in cord blood of women who demonstrated antenatal anti-SARS-CoV-2 antibodies, raising the possibility of passive immunity.
\end{abstract}

\section{Immunological assessment of SARS-CoV-2 Infection in Pregnancy}

From Diagnosis to Delivery: A multicentre prospective study 
Kate Glennon ${ }^{1}$, Jennifer Donnelly ${ }^{2}$, Susan Knowles ${ }^{3}$, Fionnuala M McAuliffe ${ }^{4}$, Alma O'Reilly ${ }^{2}$, Siobhan Corcoran $^{5}$, Jennifer Walsh ${ }^{5}$, Roger McMorrow $^{5}$, Tess Higgins $^{5}$, Lucy Bolger ${ }^{5}$, Susan Clinton ${ }^{5}$, Sarah O'Riordan $^{5}$, Alexander Start ${ }^{4}$, Doireann Roche ${ }^{5}$ Helena Bartels $^{6}$, Ciara Malone ${ }^{6}$, Karl McAuley ${ }^{7}$, Anthony McDermott ${ }^{7}$, Rosanna Inzitari ${ }^{7}$, Colm P.F. O'Donnell ${ }^{8}$, Fergal Malone ${ }^{2}$, Shane Higgins ${ }^{1,5}$, Cillian De Gascun $^{9}$, Peter Doran ${ }^{7}$ and Donal J Brennan ${ }^{1,10}$

${ }^{1}$ UCD School of Medicine, National Maternity Hospital, Holles Street, Dublin 2

${ }^{2}$ RCSI School of Medicine, Rotunda Hospital, Parnell Square, Dublin 1

${ }^{3}$ Department of Microbiology, National Maternity Hospital, Holles Street, Dublin 2

${ }^{4}$ UCD Perinatal Research Centre, School of Medicine, University College Dublin, National Maternity Hospital, Dublin, Ireland

${ }^{5}$ National Maternity Hospital, Holles Street, Dublin 2

${ }^{6}$ Rotunda Hospital, Parnell Square, Dublin 1

${ }^{7}$ Clinical Research Centre, UCD School of Medicine, St Vincent's University Hospital, Dublin 4

${ }^{8}$ Neonatal Unit, UCD School of Medicine National Maternity Hospital, Holles Street, Dublin 2

${ }^{9}$ National Virus Reference Laboratory, University College Dublin, Belfield, Dublin 4

${ }^{10}$ Systems Biology Ireland, UCD School of Medicine, Belfield, Dublin 4

* Corresponding Author:

Professor Donal J Brennan

UCD School of Medicine

National Maternity Hospital

Holles Street, Dublin 2 Ireland.

E-mail: donal.brennan@ucd.ie Tel: +353-1-7164567

Running Head

Immunological assessment of SARS-CoV-2 Infection in pregnancy.

Abstract :

Objective

We profile the maternal and fetal response to SARS-CoV-2 infection in symptomatic and asymptomatic pregnant women and make an assessment of passive immunity to the neonate,

\section{Design}

Multicentre prospective study.

\section{Setting}

Dublin, Ireland

\section{Methods}

RT-PCR for viral RNA via a nasopharyngeal swab was performed using the Cobas SARS-CoV-2 6800 platform. Maternal, and fetal serological antibody response, via umbilical cord bloods, was measured using both the Elecsys ${ }^{\circledR}$ immunoassay, Abbott SARS-CoV-2 IgG Assay and the IgM Architect assay.

\section{Main outcome Measure}


Prevalence of RT PCR positive SARS-CoV-2. Assessment of IgM and IgG anti-SARS-CoV-2 serology antibodies.

\section{Results}

Ten of twenty three symptomatic women had SARS-CoV-2 RNA in a nasopharyngeal swab. Five $(5 / 23$, $21.7 \%$ ) demonstrated serological evidence of anti-SARS-CoV-2 IgG antibodies and seven $(30.4 \%, 7 / 23)$ were positive for IgM antibodies.

In the asymptomatic cohort, the prevalence of SARS-CoV-2 infection in RNA was $0.16 \%(1 / 608)$. IgG SARSCoV-2 antibodies were detected in $1.67 \%$ (10/598, 95\% CI 0.8\%-3.1\%) and IgM in $3.51 \%(21 / 598,95 \%$ CI $2 \cdot 3-5 \cdot 5 \%)$. Nine women had repeat testing between post baseline. Four $(4 / 9,44 \%)$ remained IgM positive, one IgG positive. IgG anti SARS-CoV-2 antibodies were detectable in cord bloods from babies born to five seropositive women who delivered during the study.

\section{Conclusion}

Using two independent serological assays, we present a comprehensive illustration of the antibody response to SARS-CoV-2 in pregnancy, and show a low prevalence of asymptomatic SARS-CoV-2. Transplacental migration of anti-SARS-CoV-2 antibodies was identified in cord blood of women who demonstrated antenatal anti-SARS-CoV-2 antibodies, raising the possibility of passive immunity.

\section{Tweetable abstract}

Using independent serological assays we present a comprehensive illustration of the antibody response to SARS-Co-V2 in pregnancy

\section{Introduction}

Despite swift advances in our understanding of the SARS-CoV-2 virus, much remains to be understood regarding the timing, nature and persistence of both the humoral and cellular human response. Confirmation of an antibody response in pregnant women can direct resources in maternal services but also in the management of neonates during future surges in a similar fashion that current antenatal influenza and pertussis vaccination schedules utilise the transplacental migration of antibodies to enhance the neonatal immune system (1).

In this study, we present a comprehensive profile of the temporal serological response in pregnant women and document the presence of transplacental antibodies to SARS-CoV-2.

Maternal IgG antibodies travelling across the placenta, provide vital immunity to the new-born and have been demonstrated in infants for infections such as tetanus and human papillomavirus (HPV) $(2,3)$. To date, the evidence is sparse surrounding transplacental passage of SARS-CoV-2. Initially, at the outset of the pandemic, strict measures were adopted to reduce the risk of vertical transmission to the neonate, including isolation of babies from SARS-CoV-2 positive mothers (4). Antibodies have been demonstrated in the blood of neonates born to positive mothers when tested at birth $(5,6)$ and evidence of maternal antibodies to SARS-CoV-2 within cord bloods is slowly emerging though data is sparse $(7,8)$. Further confirmation of transplacental migration of maternal anti-SARS-CoV-2 antibodies in umbilical cord blood, could suggest the possibility of passive immunity and could even direct future vaccination protocols in pregnant women.

Determining the seroprevalence of SARS-CoV-2 has largely been based on detection of viral RNA using reverse transcription polymerase chain reaction (RT-PCR). Detection rates can be affected by collection and storage of the specimen with varying results reported depending on testing of saliva, nasal, nasopharyngeal specimens or rectal $(9,10,11,12,13)$ Therefore, detection of antibodies against SARS-CoV-2 (IgM or IgG) in serum is likely to provide a more accurate estimation of the cumulative prevalence of SARS-CoV-2 in a population.

Population-based data on SARS-CoV-2 infection in pregnancy and assessment of passive immunity to the neonate, is lacking. The aim of our study was to characterise the immune response to SARS-CoV-2 in a cohort 
of symptomatic and asymptomatic pregnant women. We assessed SARS-CoV-2 in pregnancy with combination of RT-PCR and, using three independent assays, serological detection of anti-SARS-CoV-2 antibodies. In addition, we obtained umbilical cord blood samples to matched RT-PCR positive or serological positive mothers and therefore we also present evidence of transplacental passage of anti-SARS-CoV-2 antibodies.

\section{Materials and Methods}

\section{Study Design}

This is a multicentre prospective observational study conducted at two free-standing tertiary level university maternity Hospitals in Dublin, Ireland. The Rotunda Hospital and The National Maternity Hospital provide both routine obstetric care and complex tertiary referral care for the city of Dublin and their national referral catchment areas. Each hospital delivers over 8,000 babies per annum and are amongst the largest maternity hospitals in Europe.

\section{Cohort 1 Symptomatic Pregnant Women}

Women who were symptomatic of SARS-CoV-2 $(n=23)$ were asked to attend for serological assessment of antibodies. Ten of these women were RT-PCR positive (10/23, 43\%). A $5 \mathrm{ml}$ serum and $5 \mathrm{ml}$ EDTA sample was taken during their convalescence. Participants also consented to an umbilical cord sample on delivery. All samples were analysed for the presence of IgG and IgM anti-SARS- CoV-2 antibodies.

\section{Cohort 2 Asymptomatic Pregnant Women}

Following identification of the SARS-CoV-2 positive women, we proceeded to assess the prevalence in a large scale study of asymptomatic women, initiated from the $4^{\text {th }}$ May to $15^{\text {th }}$ May 2020 . Eligible participants were identified from both inpatients and outpatient clinics. Patients were screened with a questionnaire for symptoms of SARS-CoV-2 at the time of the study and excluded if they had symptoms suggestive of active and or recent infection (recent 14 days). We approached 923 women and a total of 608 consented to and had a nasopharyngeal swab analysed. Five hundred and ninety-eight of these women consented to provide a blood sample for immunological analysis. Samples collected from participants were processed immediately and stored at $-80^{\circ} \mathrm{C}$ prior to analysis.

\section{Collection of cord blood}

A $5 \mathrm{ml}$ Serum and $5 \mathrm{ml}$ EDTA venous sample was taken from the cord after the baby was delivered and the cord was clamped. These were then processed as per the maternal serological samples

Processing and analysis of Respiratory Samples

Respiratory samples were inactivated by incubation with a lysis buffer containing guanidinium thiocyanate in a biological safety cabinet for 10 minutes prior to analysis. SARS-CoV-2 RNA testing was performed on the cobas SARS-CoV-2 6800 (Roche Molecular Systems, Branchburg, NJ) in accordance with the manufacturer's instructions. A $0.6 \mathrm{~mL}$ aliquot of each sample was loaded onto the cobas 6800 where it was combined with the cobas SARS-CoV-2 master mix containing an internal RNA control primers, and probes targeting the ORF1/a nonstructural region that is specific for SARS-CoV-2 (target 1), as well as the conserved, structural protein envelope E gene that is shared by the Sarbecovirus subgenus (target 2). Results were reported by the cobas SARS-CoV-2 test as either "detected" (targets 1 and 2 detected), "presumptive positive" (target 1 not detected; target 2 detected), or "not detected."

Processing and analysis of serology

All serological samples were processed in a single laboratory (Core Laboratory in the Clinical Research Centre, University College Dublin), in a blinded fashion using three different assays on three different platforms: the electrochemiluminescence immunoassay (14) on an automated Roche platform Cobas(r) e411, and the chemiluminescent microparticle immunoassay (CMIA) (SARS-CoV-2 IgG 75 assay; Abbott Laboratories, IL, USA) on Architect i2000SR and Alinity and the chemiluminescent microparticle immunoassay 
(CMIA) (SARS-CoV-2 IgM assay; Abbott Laboratories, IL, USA) on Architect i2000SR Plasma samples were processed immediately after collection and stored at -80degC prior to analysis.

The Elecsys anti-SARS-CoV-2 serology assay is a sandwich immunoassay intended for the detection of IgM and IgG antibodies to SARS-CoV-2 in human serum and plasma. $140 \mu \mathrm{L}(20 \mathrm{uL}+120 \mathrm{uL}$ dead volume) of sample was used in the assay. Results were determined automatically by the software by comparing the electrochemiluminescence signal obtained from the reaction product of the sample with the signal of the cut-off value previously obtained by calibration with ACOV2 Cal1 containing human serum, non-reactive for anti-SARS-CoV-2 antibodies, and ACOV Cal2 containing human serum reactive for anti-SARS-CoV-2 antibodies.

Plasma samples were also run on the Abbott Architect i2000SR and the new Alinity instruments using the Abbott SARS-CoV-2 IgG assay. The assay is a two-step immunoassay using CMIA technology for qualitative detection of IgG in human serum or plasma, raised against the nucleocapsid protein of SARS-CoV-2. The Architect requires a volume of $75 \mu \mathrm{L}$ of serum or plasma $(25 \mathrm{uL}+50 \mathrm{uL}$ dead volume). Qualitative results and index values reported by the instrument were used for analysis (15). A signal/cut-off (S/CO) ratio of [?]1.4 was interpreted as reactive. Calibration was performed and positive quality control S/CO 1.65-8.40 and negative quality control S/CO [?] 0.78 were fulfilled prior to analyses of patient samples. Within-day imprecision assessment was performed using QC material.

IgM Plasma samples were run on the Abbott Architect i2000SR. A signal/cut-off (S/CO) ratio of [?]1.0 was interpreted as reactive.

\section{Statistical Analyses}

At the prevalence study (asymptomatic cohort) design stage, it was estimated that the asymptomatic prevalence of SARS-Co-V-2 would likely range between 15 and 20\% (17). The sensitivity of current RT-PCR assays for SARS-CoV-2 has not been published but was expected to range from 70-90\%. Specificity was expected to be above $90 \%$. Therefore, to adequately power a study with a $10 \%$ prevalence rate would require between 355 and 574 patients, while if prevalence was found to be $20 \%$ this study would require between 462 and 733 patients. Median and interquartile range (IQR) are calculated for continuous variables, while counts and percentages are used to describe categorical variables. The Clopper-Pearson interval was used to find a $95 \%$ confidence interval for the probability of having positive seroprevalence results.

\section{Results}

We established two cohorts of pregnant women in whom serological assessment of both IgG and IgM antibody response to SARS-CoV-2 was assessed. In total, between both cohorts, for IgM anti SARS-CoV-2 antibodies were detected in 28 women and $\operatorname{IgG}$ antibodies were detected in 15 women. Umbilical samples were obtained from seventy-eight of these women during our study period. Nine women returned for longitudinal assessment of antibody response on days 101-122 post baseline testing.

\section{Cohort 1}

Cohort 1 consists of 23 symptomatic women, ten of whom were confirmed to be SARS-CoV-2 infected via RTPCR (Table S1). Antibody testing for IgG and IgM anti SARS-CoV-2 was conducted at various points during convalescence from day 0 to day 66 following a positive swab result. Anti-SARS-CoV-2 IgG antibodies were detected in 50\% (5/10) of the RT-PCR positive SARS-CoV-2 women using the Roche and Abbot Architect platforms. Anti-SARS-CoV-2 IgM was detectable in seven women in the symptomatic cohort, one of whom was both RT-PCR negative on nasopharyngeal swab and IgG negative in serum (Supplementary table S3b). However, in view of this, it is possible that this may more likely reflect a false positive IgM. One woman did not mount an antibody response, when tested at day 10 . She described very mild symptoms, presenting with an uncomplicated pyrexia and no respiratory symptoms.

The earliest evidence of a maternal IgG and IgM antibody response after a positive nasopharyngeal swab was at day 0 . However, this patient reported first onset of symptoms 14 days prior to her nasal RT-PCR 
testing (patient c19-13, Figure 1). Therefore, we determined that the that the earliest response between swab positive (at symptom onset) and a demonstrably IgG antibody response was at day 4 (Patient c19-12, figure 1). Detectable antibody levels were not present in women tested at day 37 and 66 post a positive nasopharyngeal swab (Supplementary table S1).

\section{Cohort 2: RT PCR testing}

Of 608 asymptomatic women who had a nasopharyngeal swab, SARS-CoV-2 RNA was detected in one woman, suggesting a prevalence of asymptomatic infection of $0 \cdot 16 \%(1 / 608,95 \%$ CI $0 \%-0.9 \%)$. Serology demonstrated positive for IgG SARS-CoV-2 antibodies but was negative for IgM antibodies. SARS-CoV-2 RNA was not detected in a nasopharyngeal swab from her baby. The umbilical cord blood, however, was positive for the presence of IgG anti-SARS-CoV-2 antibodies.

\section{Cohort 2: Serological assessment in asymptomatic pregnant women}

Samples were available for serological analysis in 598 women in the asymptomatic cohort. Anti-SARS-CoV-2 IgG antibodies were detected in 12 women using the Roche platform and ten women using the Alinity and Architect platform (Table S2). The seroprevalence rate using two independent assays was 1.67\% (10/598 95\% CI 0.8-3.1\%). Anti-SARS-CoV-2 IgM antibodies were observed in twenty-one asymptomatic women $(21 / 598$ $3.51 \%$ CI 2.3-5.5\%) (Supplementary table S3a). Of these 21 women, 8 were also IgG positive $(8 / 21,38 \%$ ) using both the Roche, Architect and Alinity platforms. Th majority of women who were antibody positive were $>24$ weeks gestation. Demographics of all women are presented in supplementary table S4

Nine women from the asymptomatic cohort returned for follow up antibody testing between 101-122 days after the baseline test (Table 1$)$. Four $(4 / 9,44 \%)$ remained IgM positive (Figure 2a). One woman was IgM positive only at baseline testing. Her follow up IgM antibodies were negative. In view of this, and the initial negative PCR and IgG anti SARS-CoV-2, it is likely that this also represents a false positive IgM result. The majority $(6 / 7,85.7 \%)$ of the seven women who were IgG positive at baseline no longer exhibited anti SARS-CoV-2 IgG antibodies (Figure 2b). Retrospective histories taken at follow-up suggests many of these women may have been mildly symptomatic at the time of the initial study

\section{Umbilical cord blood analysis}

Seventy-eight umbilical cord bloods, from mother who participated in this study, were assessed for the presence of SARS-CoV-2 antibodies. Of these, five mothers (3 in the symptomatic cohort and 2 in the asymptomatic cohort) delivered during this study period. All five umbilical cord bloods demonstrated IgG antibodies to SARS-CoV-2 (Table 2). The median antibody index was 4.88 (Roche) and 2.33 using the Alinity platform. Predictably, all of the cord bloods were negative for the presence of IgM antibodies

\section{Discussion}

\section{Main Findings}

We present a comprehensive profile of the antibody responses to SARS-CoV-2 in both symptomatic and asymptomatic pregnant woman. Our finding of anti-SARS-CoV-2 IgG antibodies in umbilical cord blood raises the possibility that passive immunity was established in babies born to mothers with a history of SARSCoV-2 infection. Our longitudinal analysis reveals key temporal features and adds additional information about the course of the virus in pregnancy.

\section{Study Strengths}

One of the major strengths of our study is the use of two independent assays to confirm seroprevalence and antibody levels. The Elecsys Anti-SARS-CoV-2 assay uses a recombinant protein representing the nucleocapsid (N) antigen for the determination of antibodies against SARS-CoV-2 (14). The Abbot SARS-CoV-2 IgG assay is a chemiluminescent microparticle immunoassay (CMIA) intended for the qualitative detection of IgG antibodies to SARS-CoV-2, which also targets the nucleocapsid protein. Both tests enable a comprehensive 
determination of the immune reaction to SARS-CoV-2 and provides reliable sensitivity and specificity (14, $15)$.

Our demonstration of anti- SARS-CoV-2 IgG antibodies in five umbilical cord samples taken in women who had confirmed evidence of infection, is noteworthy and confirms transplacental passage of anti-SARS-CoV-2 antibodies and raises the possibility of the presence of passive immunity.

Transplacental passage of SARS-CoV-2 RNA has not been demonstrated $(16,17,18,19)$, a finding consistent with previous experience in SARS and MERS (20), however our demonstration of transplacental passage of IgG antibodies could potentially play an important role in future vaccination strategies. To date, there is limited evidence of IgG presence in cord blood. The SARS-CoV-2 genome was detected in one umbilical cord in a study from Lombardy Italy and IgG in one cord sample reported from Wuhan China $(7,8)$.

Interestingly, IgG antibody persisted and was present in an umbilical cord blood sample 66 days post diagnosis of maternal SARS-CoV-2 suggesting transplacental passage of antibodies even when maternal antibodies have waned. While the maternal blood sample no longer demonstrated the presence of antibodies, the cord blood was positive for IgG anti SARS-CoV-2. (patient c19 31, Table 2). Transplacental migration of IgG antibodies begin from 13 weeks and peak in the second and third trimester (21), clearly demonstrated by studies of antenatal influenza vaccination where cord blood antibody levels are significantly higher when the mother is vaccinated in either trimesters 2 or $3(23,24)$ The efficiency of IgG transfer can vary from one antigen-specificity to another. In normal pregnancy, the transfer efficiency of IgG against pertussis can be up to $200 \%$ whereas for group B streptococcus it is only $70 \%$ (22). Our findings suggest high efficiency of transfer of IgG in the novel SARS-CoV-2, however larger cohorts will be required to substantiate these findings.

These data may help direct future antenatal vaccination programs. Antibodies have been detected in cord bloods of up to $80 \%$ of babies born to mothers who participated in a vaccination schedule (25). Maternal vaccination in pregnancy can therefore enhance passive antibody transfer as the neonatal immune system begins to mature. Knowledge of anti-SARS-CoV-2 cord blood antibody levels could help guide any future vaccination protocol in pregnancy.

Serological assessment of pregnant women may provide a more accurate assessment of seroprevalence. A study from Philadelphia demonstrated a seropositivity rate of $6.2 \%(80 / 1293)$ in a pregnant population. This was considerably higher than the estimated infection rate of $1.4 \%$ in that areas general population (27). We detected a very low prevalence rate from RT-PCR alone (1/608, 0.16\%, 95\% CI 0\%-0.9\%). However, in keeping with other published reports, our study of asymptomatic women also demonstrates a very low serological prevalence SARS-CoV-2(28). Whilst the seropositivity rate of IgM was almost three times higher than that of $\operatorname{IgG}$ anti-SARS-CoV-2 antibodies $(\mathrm{IgM}=21 / 598,3.51 \%$ and $\mathrm{IgG}=10 / 598,1.67 \%)$ both were low within our asymptomatic population.

An assessment of the temporal response in pregnancy suggests that IgM antibodies persisted for up to 122 days post baseline testing (Table 1) while IgG was no longer detectable in the majority. In other longitudinal studies of antibodies in a non-pregnant populations, IgM levels decreased rapidly in recovered patients (29). Our pregnant population were asymptomatic or exhibited mild symptoms only. In previous SARS pandemics, IgM antibodies to SARS-CoV persist for a much shorter period of time and detectable IgG antibodies and neutralizing viral antibodies persisted for up to 720 days $(30,31)$. Overall the longevity of the immune response to SARS-CoV-2 is unknown. In SARS-CoV-1 infected patients, $90 \%$ and $50 \%$ have been shown to maintain IgG antibodies for two and three years respectively (32).

Study limitation.

The asymptomatic prevalence study was implemented nine weeks after the first confirmed case of SARSCoV-2 infection was reported in Ireland (March $1^{\text {st }} 2020$ ) and 16 days after the peak of the population infection (33). Our study commenced as the rate of new cases of infection was already falling and therefore may account, in part, for the low prevalence of PCR positive cases in this cohort. However, the low antibody 
prevalence in our study correlates with other large population-based studies and suggests that even at this stage of the pandemic, the vast majority of the pregnant population remains immunologically naive.

Five seropositive patients delivered during this study period. Further analysis of the transplacental passage of antibodies in umbilical cord blood from additional women, will provide further credence to our findings and is required to assist in counselling pregnant women and directing future vaccination strategies.

\section{Conclusion}

Large scale and comprehensive assessment of the IgG and IgM antibody response to SARS-CoV-2 is vital to determine the aetiology of the virus within the pregnant population. Further analysis can confirm the transplacental transmission of anti-SARS-CoV-2 antibodies and characterize the maternal temporal response. This information could inform future public health policy regarding antenatal immunisation programs and neonatal care.

Disclosure of interests: The authors have no disclosure of interests.

\section{Contribution to authorship :}

Study Design and Conception : DB KG, SK, JD

Study Planning DB, KG, FM, SH, RM , SC, JW SK JD

Recruitment: LB, DR, SC, SOR, AS, HB, CM, KG, DB, F McA,

Analysis: K McA, AD, RI, PD, CDG, DB, KG, AOR, TH

Manuscript, SC, JW, RM, COD, SH, FM , F Mc A, KG, RI, CDG, PD, DB

Details of ethics approval : This study was approved by the ethics board of the National Maternity Hospital Holles Street Dublin on $23^{\text {rd }}$ March (Ref EC09 2020) and the Rotunda Hospital Dublin on $11^{\text {th }}$ May 2020 (REC 2020-013)

\section{Funding}

This research did not receive any specific grant from funding agencies in the public, commercial, or not-forprofit sectors. It was supported by the UCD Clinical Research Centre in the UCD School of Medicine.

\section{Acknowledgements}

We would like to acknowledge and thank the women who participated in this study

We would also like to thank the following who contributed to recruitment, sample collection and data entry:

Dr Grace Ryan, Dr Max Waterstone, Ms Julia Dirk, Dr Josh Birrane, Dr Niamh Keating, Dr Michael Wilkinson, Dr Catherine McNestry, Dr Vinita Vince, Dr Moni Hersi, Dr Alex Dakin, Dr Maebh Horan, Dr Catriona Wynn, Dr Emma Tuthill, Dr Maria Cheung, Dr Marguerite O'Brien, Dr Sahr Yambasu, Dr Katrina Vandamme, Dr Miriam Poulton, Dr Aliya AlSadani, Dr Fiona O Toole, Dr Rawia Ahmed, Dr Claire McCarthy, Dr Claire O'Connor, Ms Lucy Murphy Ms Ashwini deSouza Ms Emma Gorman We would also like to acknowledge all of our midwifery colleagues particularly Ms Caroline Brophy, Ms Val Kinsella, Ms Helen McCabe, Ms Martina Cronin and all midwifery on delivery unit in NMH who helped collect cord bloods. Our midwifery colleagues in the Rotunda Hospital, Ms Kathleen Kelly, Ms Cianna Kenny, Ms Siobhan Ryan, Ms Orlaith Stone, Ms Hilary Giligan, - senior midwifery support from Ms Fiona Hanrahan, Ms Ger Gannon, Ms Anne Marie Sliney. We would also like to thank Ms Marie Culliton for technical support in NMH laboratory, Ms Brenda Molloy for her help in developing the study database and Ms Athina Krinidi for statistical support.

\section{References}

1. Kong KL, Krishnaswamy S, Giles ML. Maternal vaccinations. Aust J Gen Pract. 2020;49(10):630-5. 
2. Fu C, Lu L, Wu H, Shaman J, Cao Y, Fang F, et al. Placental antibody transfer efficiency and maternal levels: specific for measles, coxsackievirus A16, enterovirus 71, poliomyelitis I-III and HIV-1 antibodies. Sci Rep. 2016;6:38874.

3. Albrecht M, Arck PC. Vertically Transferred Immunity in Neonates: Mothers, Mechanisms and Mediators. Front Immunol. 2020;11:555.

4. Wang X, Zhou Z, Zhang J, Zhu F, Tang Y, Shen X. A case of 2019 Novel Coronavirus in a pregnant woman with preterm delivery. Clin Infect Dis. 2020.

5. Zeng H, Xu C, Fan J, Tang Y, Deng Q, Zhang W, et al. Antibodies in Infants Born to Mothers With COVID-19 Pneumonia. Jama. 2020;323(18):1848-9.

6. Dong L, Tian J, He S, Zhu C, Wang J, Liu C, et al. Possible Vertical Transmission of SARS-CoV-2 From an Infected Mother to Her Newborn. Jama. 2020;323(18):1846-8.

7. Gao X, Wang S, Zeng W, Chen S, Wu J, Lin X, Liu Y, Sun Z, Feng L. Clinical and immunologic features among COVID-19-affected mother-infant pairs: antibodies to SARS-CoV-2 detected in breast milk. New Microbes New Infect. 2020 Sep;37:100752. doi: 10.1016/j.nmni.2020.100752. Epub 2020 Sep 1.

8. Fenizia C, Biasin M, Cetin I, Vergani P, Mileto D, Spinillo A, et al. Analysis of SARS-CoV-2 vertical transmission during pregnancy. Nat Commun. 2020;11(1):5128.

9. Azzi L, Baj A, Alberio T, Lualdi M, Veronesi G, Carcano G, et al. Rapid Salivary Test suitable for a mass screening program to detect SARS-CoV-2: A diagnostic accuracy study. J Infect. 2020;81(3):e75-e8.

10. Basu A, Zinger T, Inglima K, Woo KM, Atie O, Yurasits L, et al. Performance of Abbott ID Now COVID19 Rapid Nucleic Acid Amplification Test Using Nasopharyngeal Swabs Transported in Viral Transport Media and Dry Nasal Swabs in a New York City Academic Institution. J Clin Microbiol. 2020;58(8).

11. Fernandes LL, Pacheco VB, Borges L, Athwal HK, de Paula Eduardo F, Bezinelli L, et al. Saliva in the Diagnosis of COVID-19: A Review and New Research Directions. J Dent Res. 2020:22034520960070.

12. Cheuk S, Wong Y, Tse H, Siu HK, Kwong TS, Chu MY, et al. Posterior oropharyngeal saliva for the detection of SARS-CoV-2. Clin Infect Dis. 2020.

13. Xu Y, Li X, Zhu B, Liang H, Fang C, Gong Y, et al. Characteristics of pediatric SARS-CoV-2 infection and potential evidence for persistent fecal viral shedding. Nat Med. 2020;26(4):502-5.

14. Elecsys. Elecsys(r) Anti-SARS-CoV-2. Package Insert 2020-04, V1.0; Material Numbers 09203095190 and 0920307919

15. Bryan A, Pepper G, Wener MH, Fink SL, Morishima C, Chaudhary A, et al. Performance Characteristics of the Abbott Architect SARS-CoV-2 IgG Assay and Seroprevalence in Boise, Idaho. J Clin Microbiol. 2020.

16. Lamouroux A, Attie-Bitach T, Martinovic J, Leruez-Ville M, Ville Y. Evidence for and against vertical transmission for SARS-CoV-2 (COVID-19). Am J Obstet Gynecol. 2020.

17. Chen H, Guo J, Wang C, Luo F, Yu X, Zhang W, et al. Clinical characteristics and intrauterine vertical transmission potential of COVID-19 infection in nine pregnant women: a retrospective review of medical records. Lancet. 2020;395(10226):809-15.

18. Schwartz DA. An Analysis of 38 Pregnant Women with COVID-19, Their Newborn Infants, and Maternal-Fetal Transmission of SARS-CoV-2: Maternal Coronavirus Infections and Pregnancy Outcomes. Arch Pathol Lab Med. 2020.

19. Rasmussen SA, Smulian JC, Lednicky JA, Wen TS, Jamieson DJ. Coronavirus Disease 2019 (COVID-19) and pregnancy: what obstetricians need to know. Am J Obstet Gynecol. 2020 May;222(5):415-426. doi: 10.1016/j.ajog.2020.02.017. Epub 2020 Feb 24. PMID: 32105680; PMCID: PMC7093856. 
20. Malek A, Sager R, Kuhn P, Nicolaides KH, Schneider H. Evolution of maternofetal transport of immunoglobulins during human pregnancy. Am J Reprod Immunol. 1996;36(5):248-55.

21. Fouda GG, Martinez DR, Swamy GK, Permar SR. The Impact of IgG transplacental transfer on early life immunity. Immunohorizons. 2018;2(1):14-25.

22. Zhong Z, Haltalli M, Holder B, Rice T, Donaldson B, O'Driscoll M, et al. The impact of timing of maternal influenza immunization on infant antibody levels at birth. Clin Exp Immunol. 2019;195(2):139-52.

23. Kohler PF, Farr RS. Elevation of cord over maternal IgG immunoglobulin: evidence for an active placental IgG transport. Nature. 1966;210(5040):1070-1.

24. Puleston RL, Bugg G, Hoschler K, Konje J, Thornton J, Stephenson I, et al. Observational study to investigate vertically acquired passive immunity in babies of mothers vaccinated against H1N1v during pregnancy. Health Technol Assess. 2010;14(55):1-82.

25. Ochola R, Sande C, Fegan G, Scott PD, Medley GF, Cane PA, et al. The level and duration of RSV-specific maternal IgG in infants in Kilifi Kenya. PLoS One. 2009;4(12):e8088.

26. Cosma S, Borella F, Carosso A, Sciarrone A, Cusato J, Corcione S, et al. The "scar" of a pandemic: cumulative incidence of COVID-19 during the first trimester of pregnancy. J Med Virol. 2020.

27. Hou H, Wang T, Zhang B, Luo Y, Mao L, Wang F, et al. Detection of IgM and IgG antibodies in patients with coronavirus disease 2019. Clin Transl Immunology. 2020;9(5):e01136.

28. Flannery DD, Gouma S, Dhudasia MB, Mukhopadhyay S, Pfeifer MR, Woodford EC, et al. SARS-CoV-2 seroprevalence among parturient women in Philadelphia. Sci Immunol. 2020;5(49).

29. Mo H, Zeng G, Ren X, Li H, Ke C, Tan Y, et al. Longitudinal profile of antibodies against SARScoronavirus in SARS patients and their clinical significance. Respirology. 2006;11(1):49-53.

30. Shi Y, Wan Z, Li L, Li P, Li C, Ma Q, et al. Antibody responses against SARS-coronavirus and its nucleocaspid in SARS patients. J Clin Virol. 2004;31(1):66-8.

31. Wu LP, Wang NC, Chang YH, Tian XY, Na DY, Zhang LY, et al. Duration of antibody responses after severe acute respiratory syndrome. Emerg Infect Dis. 2007;13(10):1562-4.

32. Darren L. Covid 19 in Ireland. Oireachtas library and research service; 2020.

Figures and Tables

Figure 1

Graph of IgG and IgM antibody response - days between symptoms and antibody test

Hosted file

image1.emf available at https://authorea.com/users/376274/articles/493293-immunologicalassessment-of-sars-cov-2-infection-in-pregnancy-from-diagnosis-to-delivery-a-

multicentre-prospective-observational-study

Figure 2a: IgM Baseline and Follow up results

\section{Hosted file}

image2.emf available at https://authorea.com/users/376274/articles/493293-immunologicalassessment-of-sars-cov-2-infection-in-pregnancy-from-diagnosis-to-delivery-amulticentre-prospective-observational-study

Figure 2b: IgG Baseline and Follow up result

\section{Hosted file}


image3.emf available at https://authorea.com/users/376274/articles/493293-immunologicalassessment-of-sars-cov-2-infection-in-pregnancy-from-diagnosis-to-delivery-amulticentre-prospective-observational-study

Table 1: Follow up Serology IgM and IgG

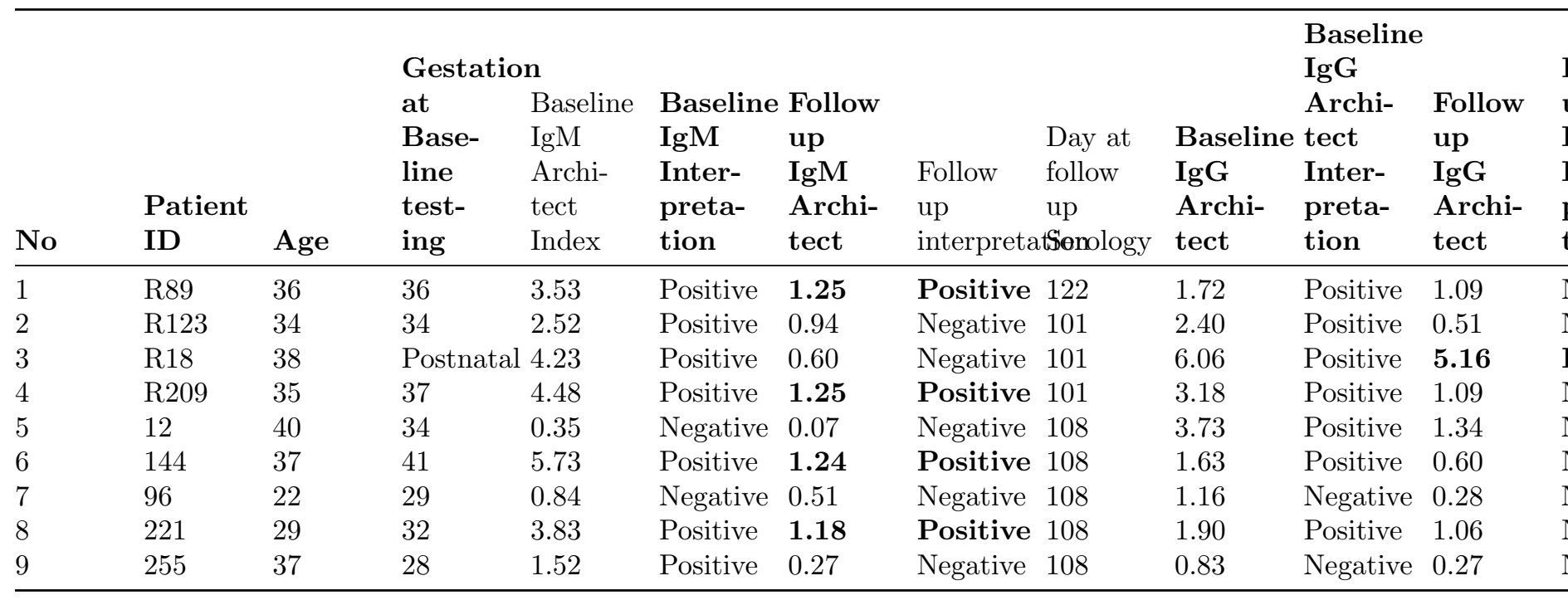

Nine asymptomatic patients returned for follow up bloods between day 108 -115 days post Baseline bloods IgG One of the seven IgG positive (Architect platform) women $(1 / 7,14.2 \%$ ) remained IgG positive on follow up

IgM Four of the nine IgM positive women $(4 / 9,44 \%)$ remained IgM positive on follow up

Table 2: Umbilical cord bloods samples positive for SARS-Co-V2

Positive umbilical cord bloods: RT-PCR Swab and antibody results

\begin{tabular}{lllll}
\hline Patient ID & Gestation at RT-PCR swab & Gestation at Delivery & Roche COI result & Roche interpretation \\
\hline $\mathrm{c}-10$ & 30 & 38 & 26.14 & Positive \\
$12^{*}$ & 34 & 34 & 2.66 & Positive \\
$\mathrm{c}-13$ & 36 & 39 & 21.51 & Positive \\
$\mathrm{c}-31$ & 31 & 40 & 3.31 & Positive \\
$144^{*}$ & 41 & 41 & 4.88 & Positive \\
\hline
\end{tabular}

